\title{
INFLUÊNCIA DO EL NIÑO-OSCILAÇÃO SUL NA PRECIPITAÇÃO EM MARINGÁ-PR, NO PERỈODO DE 1980 A 2016
}

\author{
Cíntia Minaki \\ Universidade Estadual de Maringá - UEM \\ Departamento de Geografia, Maringá, PR, Brasil \\ cminaki@uem.br \\ Otávio Cristiano Montanher \\ Universidade Estadual de Maringá - UEM \\ Departamento de Geografia, Maringá, PR, Brasil \\ otaviocmontanher@yahoo.com.br
}

\begin{abstract}
RESUMO
O El Niño-Oscilação Sul (ENOS) influencia o clima de diferentes regiões do mundo, das quais o Sul do Brasil é um exemplo. Assim, buscou-se compreender a influência do ENOS nas anomalias pluviométricas do município de Maringá-PR, localizado na Mesorregião Norte Central Paranaense. Com a série de dados mensais de precipitação, de 1980 a 2016, da Estação Climatológica Principal de Maringá (ECPM), calculou-se as anomalias locais de chuva. Utilizou-se também os dados do Índice Oscilação Sul (IOS) e da Temperatura da Superfície do Mar (TSM), divulgados pela National Oceanic and Atmospheric Administration (NOAA). Para compreender se as anomalias de chuva podem ser explicadas pelo ENOS, com base no IOS, aplicou-se as correlações de Pearson ( $r$ ) e de Kendall ( $T$ ). Aplicou-se também testes de mediana entre as anomalias de precipitação de três grupos: meses com influência do El Niño, La Niña e em condições neutras. Os resultados indicaram meses do outono e da primavera como os de maior correlação entre as variáveis, e a defasagem temporal variou de zero a cinco meses entre os coeficientes utilizados. Os testes estatísticos demonstraram a anomalia positiva de chuva em meses de EI Niño, não sendo possível concluir o inverso, na mesma intensidade, para o La Niña.
\end{abstract}

Palavras-chave: Anomalia de chuva. Índice Oscilação Sul (IOS). Temperatura da Superfície do Mar (TSM). Correlação.

\section{EL NIÑO-SOUTHERN OSCILLATION INFLUENCE ON THE PRECIPITATION IN MARINGÁ-PR, BETWEEN 1980 AND 2016}

\begin{abstract}
The El Niño-Southern Oscillation (ENSO) affects the climate from different regions of the world which the Southern Brazil is an example. So, it was aimed to understand the ENSO influence on precipitation anomalies in the municipality of Maringá-PR, placed in Central North region of the Paraná State. By using monthly data series of precipitation, from 1980 to 2016, collected in the Principal Climatological Station of Maringá (PCSM)/INMET, it was calculated the local rainfall anomalies. Were also used the Southern Oscillation Index (SOI) and Sea Surface Temperature (SST) data, provided by the National Oceanic and Atmospheric Administration (NOAA). To understand the relationship between precipitation anomalies and ENSO (described by the SOI), it was applied the Pearson and Kendall correlations. Were applied also median tests between three groups of precipitation anomalies: months under El Niño influence, months under La Niña Influence and months of neutral conditions. Results indicated autumn and spring months as the periods of higher correlation among the variables, and the lag time varied from zero to five months. The statistical tests demonstrated positive values of precipitation anomalies during EI Niño months, but it is not possible to conclude the inverse effect, at the same intensity, for La Niña.
\end{abstract}

Keywords: Precipitation anomaly. Southern Oscillation Index (SOI). Sea Surface Temperature (SST). Correlation.

$\begin{array}{lllll}\text { Caminhos de Geografia } & \text { Uberlândia - MG } & \text { v. 20, n. } 69 & \text { Mar/2019 } & \text { p. 266-281 Página } 266\end{array}$




\section{INTRODUÇÃO}

Dentre tantas adversidades climáticas que afetam as diferentes regiões do Brasil, o El NiñoOscilação Sul (ENOS) possui um padrão identificado de influência espacial, mas é dinamicamente assimétrico em seus efeitos e temporalidade. Embora seja considerado cíclico, não possui uma ocorrência regular (NERY et al., 2003, p. 230), constituindo a principal fonte de variabilidade climática de curto prazo nas escalas estacional e interanual no Brasil (CUNHA et al., 2000, p. 10).

O ENOS possui duas fases opostas, denominadas El Niño e La Niña. Com base na Temperatura da Superfície do Mar (TSM), a fase positiva também é reconhecida como fase quente, e a negativa consiste na fase fria. O impacto pluviométrico causado pelo ENOS varia, não sendo possível restringir o El Niño a um período chuvoso, e nem o La Niña a um período seco sem associá-los à área afetada. Na Região Sul, por exemplo, em anos com ocorrência de El Niño, as precipitações são abundantes, principalmente na primavera, por sua vez, partes das regiões Norte e Nordeste do Brasil podem passar por secas de diferentes intensidades (OLIVEIRA, 2005, p. 5).

A fundamentação do ENOS não envolve apenas a componente oceânica, representada pela TSM, variável em função da modificação da intensidade dos alísios, mas também a componente atmosférica, representada pela Oscilação Sul (OS). O Índice Oscilação Sul (IOS) mensura a pressão ao nível do mar nas regiões de Darwin (Austrália, $12^{\circ} 20^{\prime} \mathrm{S}, 130^{\circ} 52^{\prime} \mathrm{L}$ ) e Taiti (Polinésia Francesa, $\left.17^{\circ} 33^{\prime} \mathrm{S}, 149^{\circ} 31^{\prime} \mathrm{O}\right)$. Estando maior na primeira, o IOS é considerado negativo. Por sua vez, a pressão mais elevada no Taiti significa um valor de IOS positivo. Essa variação barométrica entre os oceanos Pacífico e Índico foi explicada pela Circulação de Walker e denominada OS (KOUSKY et al., 1984). A mesma afeta a circulação dos ventos alísios na região do oceano Pacífico Equatorial, e por vezes, associa-se à intensificação ou enfraquecimento dos eventos El Niño e La Niña, causando, respectivamente, o aquecimento ou o resfriamento anômalo das águas superficiais da bacia do oceano Pacífico. Durante os eventos ENOS, as anomalias são espacialmente mais abrangentes que a região do Pacífico Equatorial, e pela sua larga escala de atuação, atinge várias regiões do globo (KOUSKY et al., 1984; ROPELEWISKI e HALPERT, 1987; CAVALCANTI, 1996).

Muitos autores demonstraram a correlação do ENOS com a quantidade de precipitação na Região Sul do Brasil. Cita-se Cunha et al. (2000), que abordaram os impactos causados na produção agrícola nacional do trigo e da cevada; Berlato e Fontana (2003), que apresentaram os efeitos associados ao ENOS no clima do Rio Grande do Sul, e Rao e Hada (1990) que concluíram que a precipitação no trimestre setembro-outubro-novembro no Sul do Brasil, parece ter uma correlação maior com o IOS medido ao mesmo tempo ou de um mês anterior. Teixeira e Martín-Vide (2013) estimaram que quando o IOS do trimestre dezembro-janeiro-fevereiro é elevado, a precipitação no Paraná tende a aumentar três meses depois, no trimestre março-abrilmaio. Fundamentações sobre a proporção do ENOS como forçante externa na variabilidade climática interanual e intrassazonal da Região Sul são descritas por Grimm et al. (1998), Grimm et al. (2000), Grimm (2009a) e Grimm (2009b).

Além da maior umidade em todo o estado, Ferreira (2000) observou no período de 1979 a 1998 , a redução da precipitação em anos de La Niña no Noroeste e Norte Paranaense, tal como Nery et al. (2003) identificaram maior déficit hídrico na bacia hidrográfica do rio Ivaí, na atuação da fase negativa do ENOS.

Galvani et al. (1998) correlacionaram o IOS com os desvios totais mensais da precipitação em Maringá, no período de 1976 a 1996. Os resultados mais significativos ocorreram quando relacionaram o IOS com o desvio de chuva de três meses posteriores. Assim, estes autores concluíram que o IOS relativo a janeiro refletiria no total de chuva da região, referente a abril e maio, indicando um atraso de três a quatro meses entre a identificação da anomalia na região do Pacífico Tropical e a região Norte e Noroeste do Paraná.

Maringá localiza-se na Mesorregião Norte Central Paranaense (IBGE, 1990, p. 105-106), estando em altitudes que variam aproximadamente entre 360 a $580 \mathrm{~m}$. Este município de porte populacional médio foi estudado sob o enfoque climático por Deffune (1990), Santos (1996), Silveira (2003), dentre outros autores citados anteriormente, e que compuseram importantes 
sínteses climáticas regionais, apresentando a necessidade de identificar a dinâmica das adversidades dessa natureza em Maringá. Logo, a hipótese desta pesquisa centrou-se na de que o evento El Niño é responsável pelo aumento da precipitação sobre a área, enquanto sob condições de La Niña, ocorre o inverso.

Este município é influenciado, sobretudo, pelas chuvas advindas de sistemas frontais, atuantes durante todo o ano (MONTEIRO, 1969), de convecção oriunda da região Amazônica, outros sistemas de baixa pressão formados na Depressão do Chaco (SILVEIRA, 2003) e o Jato Subtropical, não havendo efeitos topográficos significativos na determinação do clima local devido à sua característica plana. Tal característica favorece o ingresso de sistemas atmosféricos tropicais e extratropicais na área. Grimm (2009a, p. 259) destacou o regime de monção característico do norte do Sul do Brasil, ressaltando a possibilidade de significativa diferença pluviométrica entre o verão e o inverno, enquanto autores como Nimer (1989), Silveira (2003) e Maack (2012) reforçaram a transição climática entre o clima tropical típico e o subtropical.

O fato é que em anos de ocorrência de ENOS, tem-se questionado sobre o incremento do volume de chuvas, podendo haver o fortalecimento dos sistemas de baixa pressão na área, com o aumento inclusive da TSM nas águas do Atlântico Sul. Assim, buscou-se avaliar a relação entre o fenômeno ENOS e a precipitação mensal de Maringá-PR, no período de 1980 a 2016, na perspectiva de responder às seguintes perguntas: Como as variações do fenômeno ENOS, caracterizado pelo IOS, influenciam a precipitação em Maringá? Qual é a defasagem temporal desta influência? Em eventos de El Niño e de La Niña, há diferença na precipitação, quando comparada com os períodos de tempo que não estão sob influência do ENOS? Quanto mais intenso for o evento de El Niño/La Niña, maior é a diferença de precipitação com relação aos anos de neutralidade?

\section{MATERIAIS}

Utilizou-se os dados de precipitação mensal da Estação Climatológica Principal de Maringá (ECPM) / Instituto Nacional de Meteorologia (INMET), de 1980 a 2016. A ECPM localiza-se no campus principal da Universidade Estadual de Maringá (UEM) desde setembro de 1979, sob as coordenadas $23^{\circ} 24^{\prime} 19^{\prime \prime S}$ e 515'58"O e altitude de 542 metros (Figura 1). Pertence à rede de estações convencionais da Organização Meteorológica Mundial (OMM), sob o código 83767.

Utilizou-se, ainda, os dados mensais do IOS e de anomalias de TSM correspondentes à região Niño 3.4. Ambos são divulgados pela National Oceanic and Atmospheric Administration (NOAA), e foram usados para o mesmo período e escala temporal da série de precipitação

Figura 1 - Localização da área de estudo, com destaque para a ECPM.

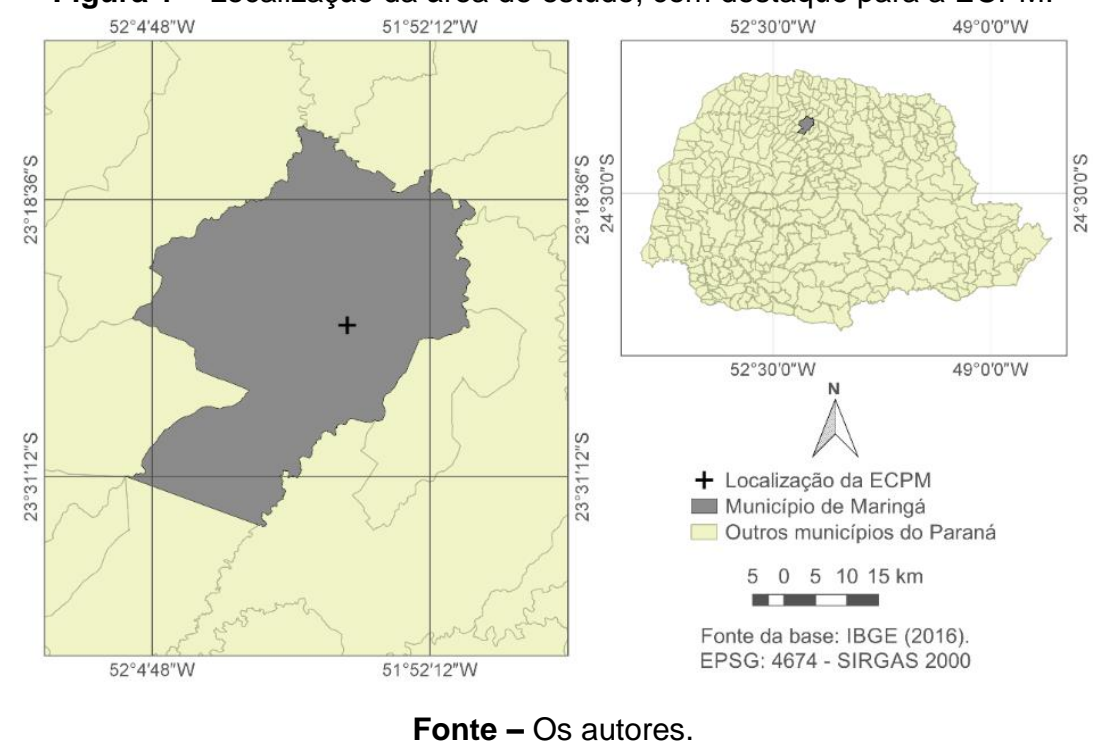




\section{MÉTODOS}

A metodologia da pesquisa estruturou-se conforme o fluxograma apresentado na Figura 2. Os dados básicos para esta pesquisa se constituem em três séries temporais: dados de precipitação, adquiridos a partir de estação climatológica convencional, e séries do IOS e de valores de anomalias de temperatura de superfície do mar. Essas três séries estão representadas na parte superior do fluxograma e os procedimentos seguem a partir delas. Na sequência, todos os passos estão descritos em maior detalhe.

Figura 2 - Estrutura da pesquisa.

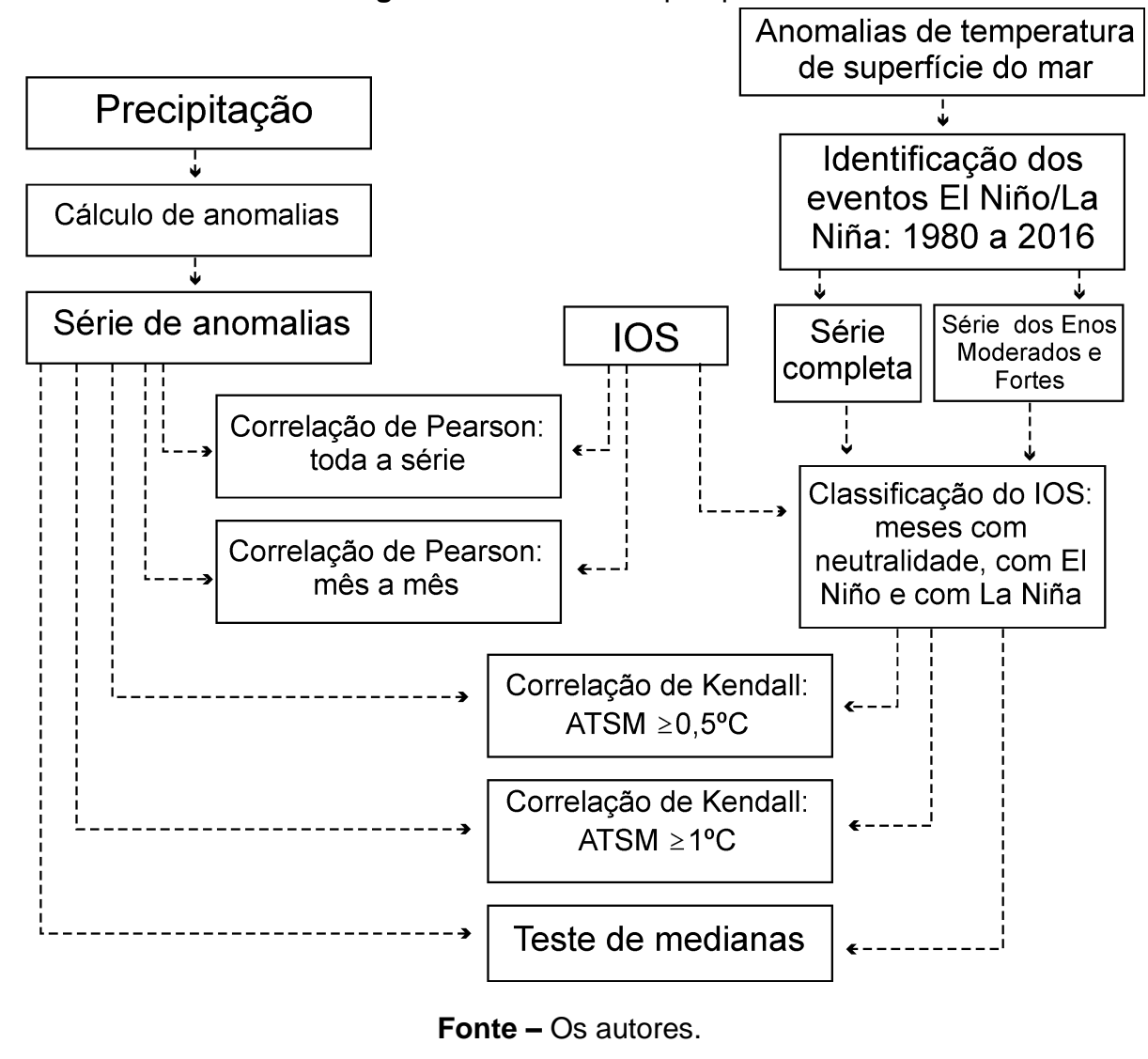

\section{CÁLCULO DE ANOMALIAS}

Inicialmente, calculou-se a anomalia de chuva do período de 1980 a 2016, associando cada valor mensal à média e desvio padrão de cada mês da série considerada (Equação 1):

$$
A_{m}=\frac{P_{m}-\overline{P_{m}}}{\sigma_{m}}
$$

Em que:

$A_{m}=$ anomalia de precipitação para um dos casos do mês $m$ (adimensional);

$P_{m}=$ precipitação de um dos casos do mês $m(\mathrm{~mm})$;

$\overline{P_{m}}=$ precipitação média para um mês $m(\mathrm{~mm})$;

$\sigma_{m}=$ desvio padrão da precipitação para um mês $m(\mathrm{~mm})$. 
O uso das anomalias descritas, ao invés dos valores absolutos de chuva, permite retirar a característica sazonal da série temporal, da mesma forma como foi feito por Galvani et al. (1998). No entanto, uma diferença entre o cálculo das anomalias deste trabalho e o supracitado é a razão pelo desvio padrão mensal, cujo objetivo foi normalizar as diferentes variâncias existentes entre os meses do ano.

\section{CORRELAÇÃO}

Com os valores das anomalias de chuva e os respectivos valores de IOS, aplicou-se a correlação de Pearson (r), tal como Galvani et al. (1998) e Teixeira e Martín-Vide (2013), utilizando-se, em um primeiro momento, todos os meses da série de 1980 a 2016 e considerando uma defasagem de 0 a 12 meses. Na sequência, a correlação foi recalculada para cada mês, com o intuito de se avaliar se há diferenças sazonais na relação entre IOS e a precipitação em Maringá.

Além do uso de todos os meses da série temporal para a correlação entre precipitação e o IOS, também foi testada a correlação entre essas duas variáveis apenas com os meses que estariam sob influência dos eventos de El Niño e de La Niña. Isso porque, quando o Oceano Pacífico está em condição de TSM próxima à média, supõe-se que outros processos atmosféricos de variabilidade interna podem estar influenciando a precipitação sobre a estação avaliada. Se houver diferença significativa entre os resultados obtidos para a correlação entre todos os meses e para as correlações obtidas para os meses sob efeito do ENOS, a hipótese é confirmada.

A separação dos valores de IOS respectivos aos eventos de El Niño / La Niña foi feita a partir de dados de série temporal de anomalias de TSM da região do Niño 3.4. Esses valores correspondem às médias móveis de três meses das anomalias de TSM. Os eventos de El Niño são definidos quando os valores dessa série são superiores a $0,5^{\circ} \mathrm{C}$, enquanto os eventos de La Niña são definidos quando as anomalias são inferiores a $-0,5^{\circ} \mathrm{C}$. Ainda, a intensidade do $\mathrm{El}$ Niño / La Niña é definida conforme classificação do Climate Prediction Center/ National Oceanic and Atmospheric Administration (CPC/NOAA), que varia a cada 0,5으 (Tabela 1).

Tabela 1 - Intensidade dos eventos El Niño e La Niña, conforme as anomalias de TSM (A) da região Niño 3.4.

\begin{tabular}{c|c|c}
\multirow{2}{*}{$\begin{array}{c}\text { Intensidade do } \\
\text { evento }\end{array}$} & \multicolumn{2}{|c}{ Intervalos de anomalias de TSM } \\
\cline { 2 - 3 } & El Niño & La Niña \\
\hline Fraco & $0,5^{\circ} \mathrm{C} \leq \mathrm{A}<1^{\circ} \mathrm{C}$ & $-0,5^{\circ} \mathrm{C} \geq \mathrm{A}>-1^{\circ} \mathrm{C}$ \\
\hline Moderado & $1^{\circ} \mathrm{C} \leq \mathrm{A}<1,5^{\circ} \mathrm{C}$ & $-1^{\circ} \mathrm{C} \geq \mathrm{A}>-1,5^{\circ} \mathrm{C}$ \\
\hline Forte & $1,5^{\circ} \mathrm{C} \leq \mathrm{A}<2^{\circ} \mathrm{C}$ & $-1,5^{\circ} \mathrm{C} \geq \mathrm{A}>-2^{\circ} \mathrm{C}$ \\
\hline Muito forte & $\mathrm{A} \geq 2^{\circ} \mathrm{C}$ & $\mathrm{A} \leq-2^{\circ} \mathrm{C}$ \\
& $\begin{array}{c}\text { Fonte }-\mathrm{CPC} / \mathrm{NOAA} . \\
\text { Organização }- \text { Os autores. }\end{array}$
\end{tabular}

A separação da série histórica em dois grupos - meses considerados "normais" devido à condição de neutralidade e os meses sob influência do ENOS -, fez com que as anomalias de precipitação tivessem uma distribuição não contínua ao longo dos valores de anomalias de TSM (Figura 3). Isso ocorre basicamente porque os valores normais são considerados entre $-0,5^{\circ} \mathrm{C}$ e $0,5^{\circ} \mathrm{C}$. Adicionalmente, se os eventos de El Niño e La Niña fracos forem desconsiderados, a distribuição fica ainda mais descontínua (Figura 3). 
Figura 3 - Distribuição entre as anomalias de precipitação e de TSM para a região Niño 3.4 do Oceano Pacífico Equatorial. Em a) são apresentados todos os meses sob influência do ENOS e em b) são apresentados apenas os meses com eventos moderados e fortes.
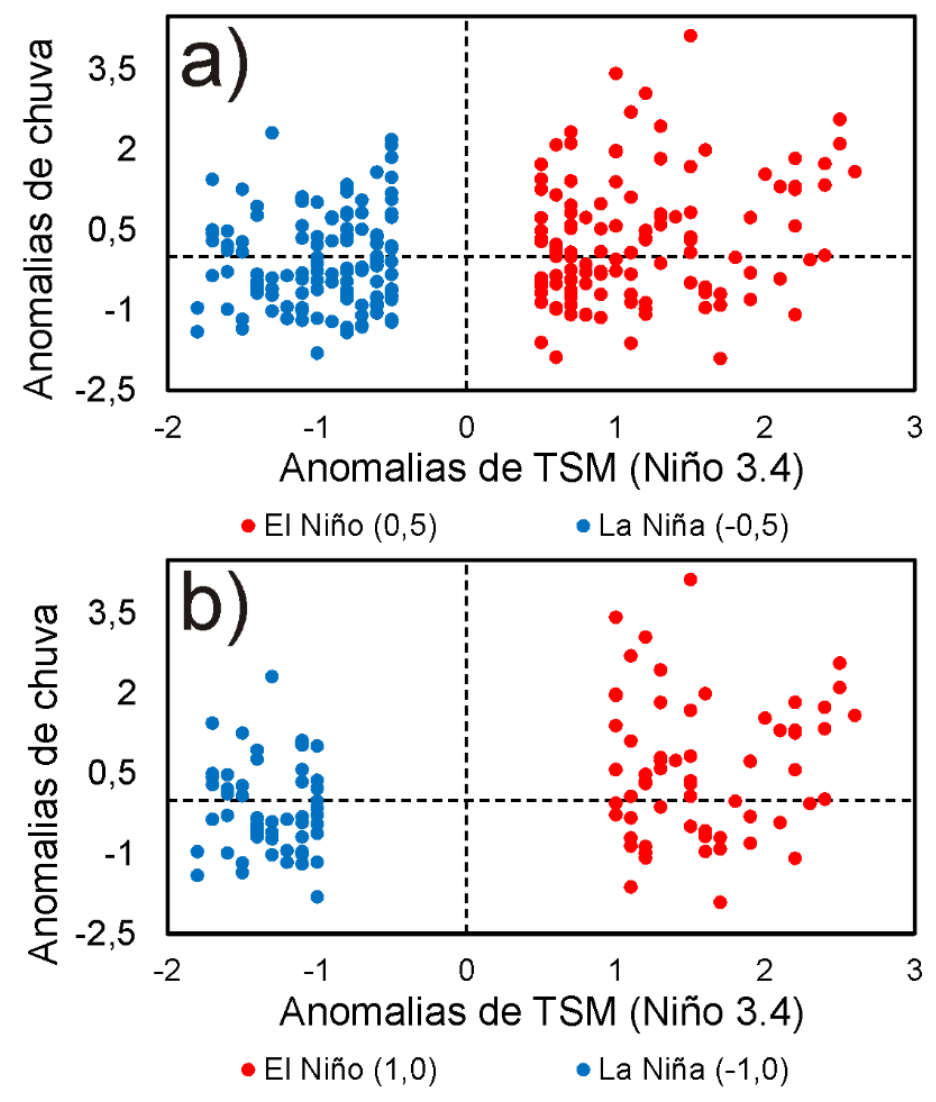

Fonte - Os autores.

Essa característica da distribuição dos dados faz com que a aplicação da correlação de Pearson seja prejudicada, pois o pressuposto de relação linearmente contínua entre as duas variáveis não é verdadeiro. Adicionalmente, como as anomalias de TSM são utilizadas para seleção dos valores de IOS, os resíduos de uma reta ajustada entre as anomalias de precipitação e o IOS não são independentes.

Como alternativa à correlação de Pearson, para a análise que separou os meses "normais" dos meses sob influência do ENOS, aplicou-se o coeficiente de correlação não-paramétrico de Kendall $(\mathrm{T})$. Foram feitas duas análises de correlação de Kendall; na primeira utilizou-se todos os meses sob influência do ENOS (módulo de anomalia de TSM $\geq 0,5^{\circ} \mathrm{C}$ ) e a segunda utilizou apenas os meses com eventos moderados ou mais fortes (módulo de anomalia de TSM $\geq 1^{\circ} \mathrm{C}$ ), conforme a Tabela 1. Realizou-se os testes de hipótese sobre os coeficientes de correlação de Pearson e de Kendall considerando o nível de significância $\alpha: 0,05$.

\section{TESTES DE MEDIANA}

As correlações descritas no tópico anterior buscaram avaliar o quanto o ENOS, representado pelo IOS, está relacionado com as anomalias de precipitação em Maringá. No entanto, embora apontem as relações gerais entre o ENOS e a precipitação, tais correlações não permitem avaliar questões mais pontuais, como, por exemplo, se as anomalias de precipitação são maiores durante os eventos de El Niño em relação aos meses normais, ou menores durante os eventos de La Niña, como sugerido pela bibliografia consultada (TRENBERTH, 1997; OLIVEIRA, 2005; TEIXEIRA e MARTÍN-VIDE, 2013). 
Com objetivo de responder essas questões com maior especificidade, buscou-se testar a diferença entre os valores de tendência central das anomalias de três grupos: meses "normais", meses sob influência do El Niño e meses sob influência do La Niña. A diferenciação dos meses entre esses três grupos foi feita com base nos valores de Anomalias da Temperatura da Superfície do Mar (ATSM) da região Niño 3.4, conforme a Tabela 1.

O teste de médias paramétrico tradicional, aplicável para esse contexto, é o teste t não-pareado. No entanto, para aplicação deste teste é necessário assumir a distribuição normal dos conjuntos amostrais. Para testar esse pressuposto foi adotado o teste Kolmogorov-Smirnov, alterado por Lilliefors, devido ao fato de que todos os conjuntos apresentam número amostral maior que 50 $(\mathrm{N}>50)$. O nível de significância adotado para o teste de normalidade foi de $\alpha: 0,05$. A avaliação preliminar dos resultados dos testes de normalidade mostrou que grande parte dos conjuntos de valores de anomalias não seguem distribuição normal. Portanto, optou-se pelo teste nãoparamétrico de Mann-Whitney para comparação de medianas. Todos os testes estatísticos foram feitos com uso do software gratuito R (R CORE TEAM, 2017).

\section{RESULTADOS E DISCUSSÕES}

\section{CORRELAÇÃO ENTRE O IOS E AS ANOMALIAS DE CHUVA}

A Figura 4 apresenta os resultados da correlação de Pearson $(R)$ entre o IOS e as anomalias de precipitação para toda a série temporal da ECPM utilizada. As correlações são predominantemente negativas, conforme esperado para a relação entre o IOS e a quantidade de chuvas (IOS negativos indicam eventos de El Niño, os quais provocam chuvas mais abundantes na região Sul do Brasil). A defasagem temporal que apresentou a maior correlação foi de dois meses, embora entre um e cinco meses as correlações sejam significativas (Figura 4). No entanto, mesmo a defasagem temporal de maior correlação (dois meses, R: -0,149), mostra que - IOS possui um baixo potencial de explicação para toda a série temporal de anomalias de chuvas ( $\left.R^{2}: 0,022\right)$.

Figura 4 - Correlação de Pearson (R) entre o IOS e as anomalias de chuva para todos os meses da série temporal. Observe que a linha contínua denota os meses em que a correlação é significativa. Número total de meses: 444.

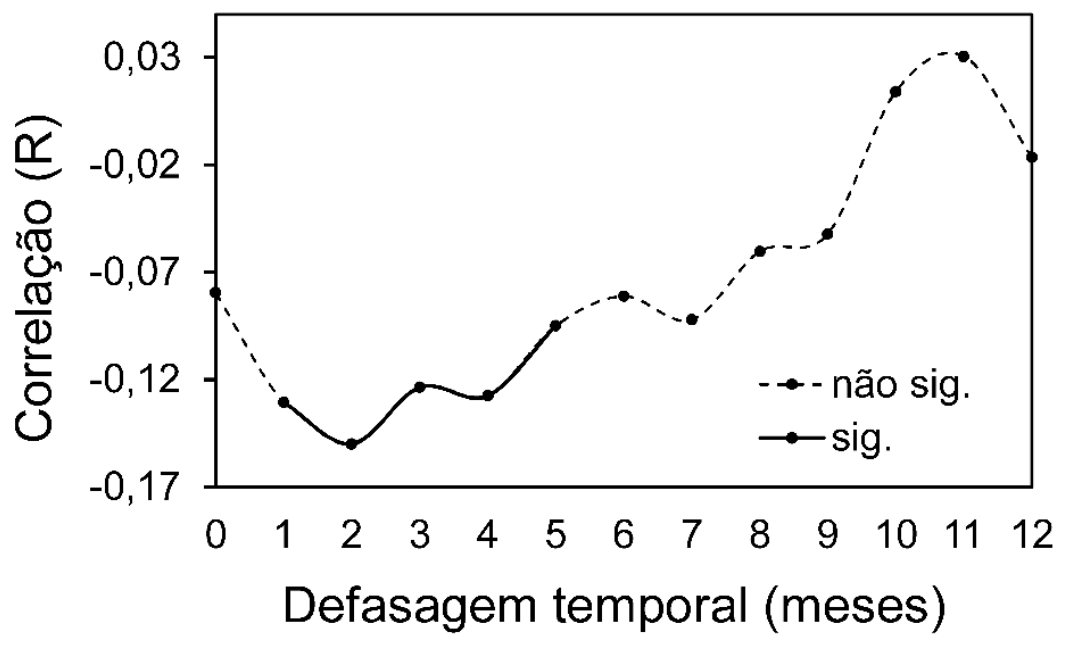

Fonte - Os autores.

Quando a correlação de Pearson é aplicada separando-se mês a mês (Figura 5), os resultados são bem diferentes daqueles observados para todo o período (Figura 4). As anomalias de chuvas de alguns meses possuem claramente uma maior correlação com o IOS do que outros. Observou-se que os meses de abril e maio (outono) e os meses de setembro, novembro e 
dezembro (principalmente os dois primeiros, primavera), apresentam correlações significativas entre as duas variáveis analisadas. Galvani et al. (1998) ao concluírem que o IOS de janeiro reflete a alteração na mesma área de estudo, três a quatro meses depois, chegaram também aos meses de abril e maio, como representativos de maior correlação. Por sua vez, Hao e Rada (1990, p. 90) questionaram a relação mais forte das chuvas de primavera com o IOS. Os sistemas frontais provavelmente bloqueados pela intensificação da célula de Hadley, permaneceriam mais tempo sobre a Região Sul do Brasil, não se deslocando para outras áreas.

O mês de abril marcou o início de intensa precipitação no Sul do Brasil, nos episódios de El Niño de 1986-1987, e nos anos de 1992 e 1993, no ENOS estendido de 1990 a 1994 (CAVALCANTI, 1996). É válido lembrar que nas estações de transição, outono e primavera, o jato subtropical posiciona-se sobre o Sul do Brasil (GRIMM, 2009a, p. 267) influenciando os valores máximos de precipitação na área e provavelmente, fortalecidos pelo evento ENOS (CAVALCANTI, 1996).

Segundo Grimm (2009b, p. 360), "a primavera é a estação mais propícia às teleconexões com o oceano Pacífico Tropical", no entanto, o efeito do ENOS na precipitação não é uniforme, uma vez que as condições atmosféricas variam ao longo do ano, variando também a propagação das ondas de Rossby, sendo em média novembro, na fase quente do evento, o mês com influências mais significativas (GRIMM et al., 1998; GRIMM, 2009b, p. 371).

Figura 5 - Correlação de Pearson entre o IOS e as anomalias de chuva, separados por cada mês. As linhas pontilhadas em 0,325 e -0,325 mostram o limite de significância do R. Número de casos em cada mês: 37. Em a) estão apresentados os meses de janeiro a junho e em b) os meses de julho a dezembro.

Observe que os meses que possuem as maiores correlações estão nas cores azul (a) e verde (b).
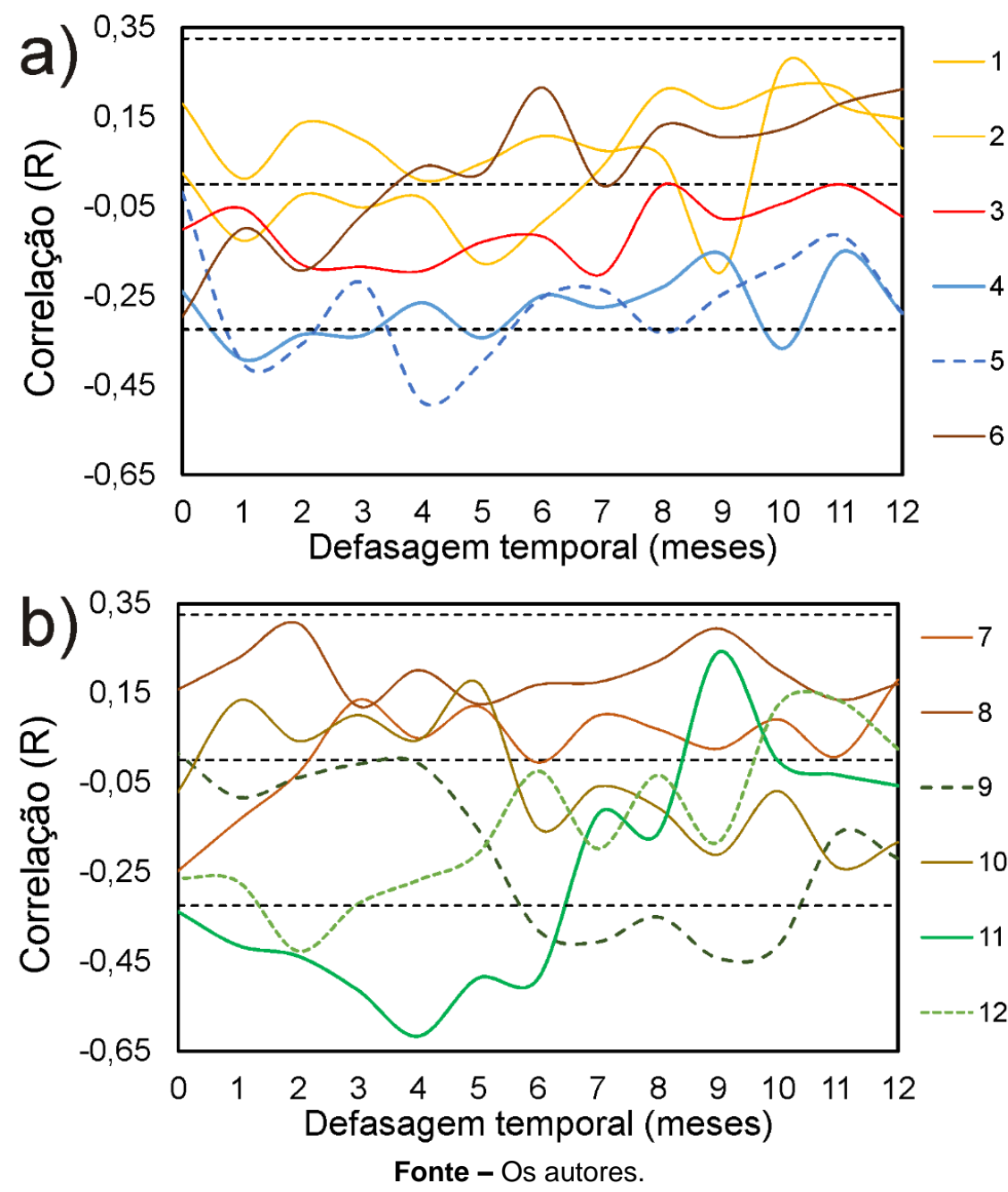
Assim como a correlação de Pearson, os resultados da aplicação do coeficiente de Kendall também apontam para relações inversamente proporcionais entre o IOS e as anomalias de precipitação (Figura 6). De modo geral, as correlações de Kendall mostram quase o mesmo período de influência dos resultados obtidos pela correlação de Pearson, com diferença de um mês. Enquanto a correlação para todos os meses mostrou uma defasagem de um a cinco meses, a correlação aplicada apenas aos meses sob influência do ENOS indica relações significativas para a defasagem de zero (simultâneo) a quatro meses. Possivelmente, quando há algum evento (El Niño / La Niña), os efeitos do Pacífico Equatorial sobre a América do Sul são sentidos com maior velocidade do que em condições normais.

Outra interpretação que pode ser feita sobre os resultados sintetizados na Figura 6 é que as correlações de Kendall são visivelmente diferentes no período em que são significativas (defasagem entre zero e quatro meses), e a partir de cinco meses possuem valores similares. Nesse período de influência dos eventos ENOS, pode-se afirmar que quando o IOS é comparado com as anomalias de precipitação para os meses de eventos moderados e fortes, há uma maior correlação entre as duas variáveis do que quando são considerados também os eventos fracos (módulos de TSM entre $0,5^{\circ} \mathrm{C}$ e $1^{\circ} \mathrm{C}$ ). Portanto, os eventos ENOS moderados e fortes têm maior relação com as anomalias de precipitação para a estação de Maringá do que os eventos fracos.

Figura 6 - Correlação de Kendall entre o IOS e as anomalias de chuva para os meses sob influência do ENOS. Observe que as linhas contínuas denotam os meses em que a correlação é significativa. Número de casos considerando todos os meses sob influência do ENOS: 242. Número de meses com eventos de ENOS moderados e fortes: 115.

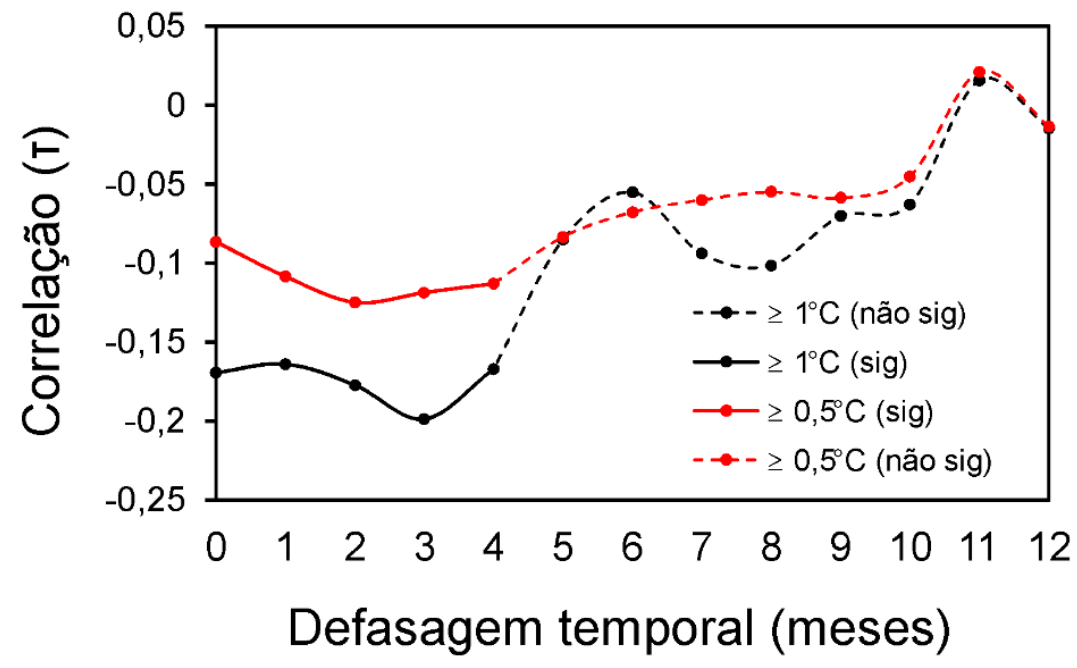

Fonte - Os autores.

A Figura 7 demonstra parte das séries temporais trabalhadas para exemplificar as relações avaliadas pelas correlações aplicadas mês a mês. Nesta representação tem-se o evento El Niño de 1982/1983, um dos mais intensos nas duas últimas décadas do século XX. Quando o IOS está elevado (valor negativo), a quantidade de chuvas não é concomitantemente alta, no entanto, observa-se que a mesma vai se elevando até atingir valores significativos acima da média. $O$ período de junho a setembro de 1982 registrou aumento do IOS e também da TSM, e posteriormente, de outubro de 1982 a janeiro de 1983, as precipitações mensais registradas foram acima da média. O mesmo se verifica com o IOS de janeiro e fevereiro de 1983 e as quantidades crescentes de chuva em abril, maio e junho do mesmo ano, configurando um inverno chuvoso. Na Figura 7, também é possível observar o aumento isolado do IOS em julho de 1983, após um mês com IOS zero, e o posterior aumento significativo da chuva em setembro do mesmo ano, sobressaindo-se aos meses de outubro, novembro e dezembro. Ao se observar um evento integral e prolongado de El Niño, como o considerado, nota-se a efetividade dos resultados das correlações, assim como outras variações menos similares, resultantes dos demais fatores que podem influenciar as anomalias de chuvas em Maringá. 
Figura 7 - Séries de IOS e de precipitação durante um evento El Niño, nos anos de 1982 a 1983. Os marcadores com preenchimento vermelho na linha do IOS destacam meses com IOS elevado ou em elevação (valor negativo).

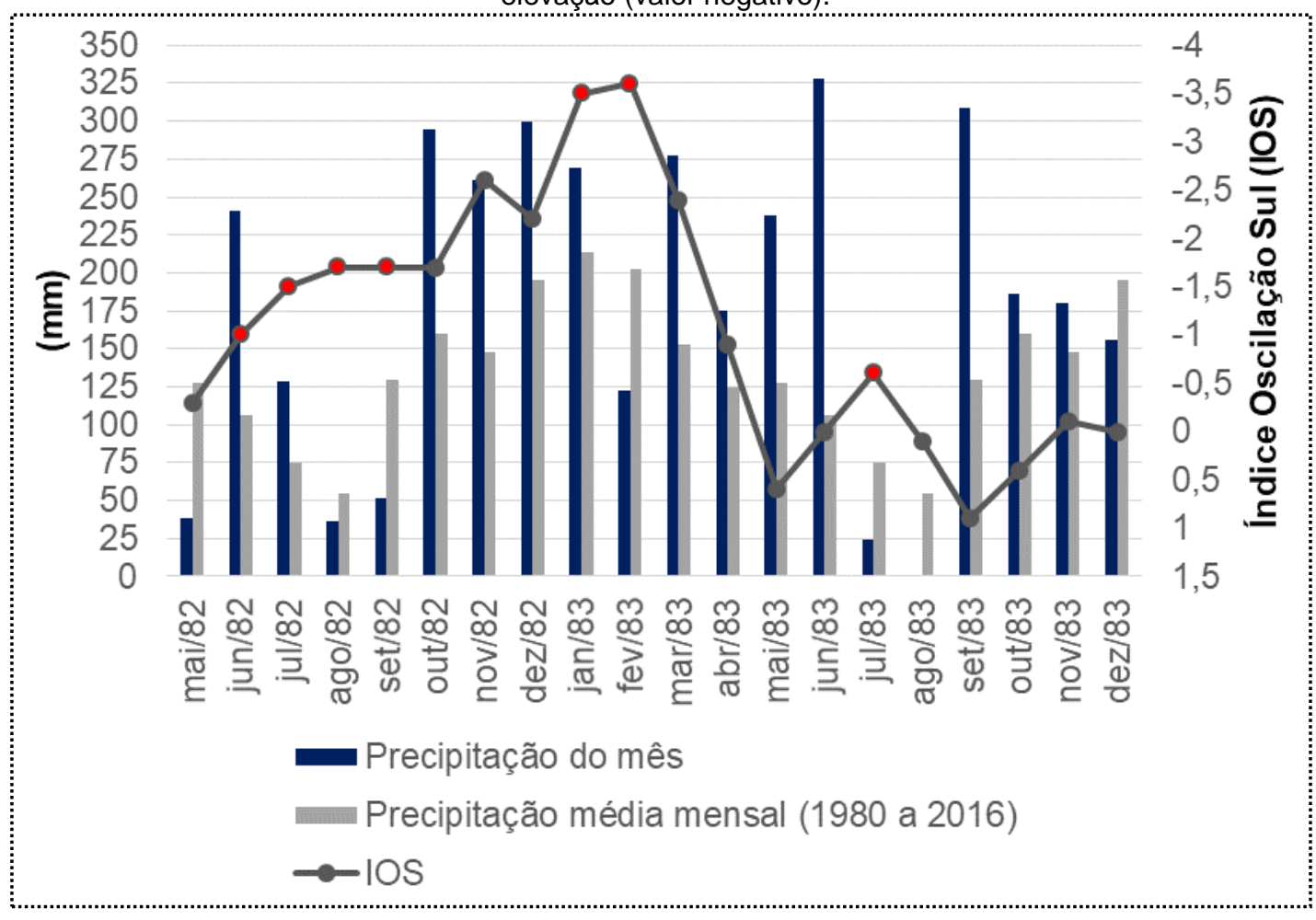

Fonte - Os autores.

\section{COMPARAÇÃO ENTRE MESES EM CONDIÇÕES NORMAIS E SOB INFLUÊNCIA DO ENOS}

As estatísticas descritivas, bem como o resultado dos testes de normalidade dos três grupos avaliados encontram-se nas Tabelas 2 (El Niño), 3 (La Niña) e 4 (meses normais). Todo o conjunto de levantamento das descritivas, bem como os testes de normalidade e medianas, foram feitos para os casos de anomalias de precipitação simultâneos aos eventos ENOS (mesmo mês) e até quatro meses de defasagem. Essa defasagem foi escolhida com base nos principais resultados obtidos a partir das correlações apresentadas no tópico anterior.

As médias de anomalias observadas na Tabela 2 mostram que, em geral, os meses que estão sob influência do El Niño têm chuvas mais abundantes. Quando apenas os eventos moderados e fortes são considerados, as médias são ainda maiores. Uma tendência aproximada de diminuição das médias conforme aumenta-se a defasagem (Tabela 2), sugere que os eventos de El Niño possuem uma resposta rápida com relação à precipitação em Maringá.

Por sua vez, as médias de anomalias observadas na Tabela 3 mostram que, em geral, os meses que estão sob influência do La Niña têm chuvas menos abundantes. Apesar de os meses em condições neutras também possuírem médias negativas de anomalias (Tabela 4), os valores são mais próximos de zero do que aqueles observados na Tabela 3 (La Niña). Assim como para os eventos de El Niño, quando apenas os eventos moderados e fortes de La Niña são levados em conta, os valores médios são mais distantes de zero do que quando são contabilizados os eventos de intensidade fraca. Por outro lado, ao contrário das médias observadas das anomalias sob efeito do EI Niño, as anomalias de precipitação sob efeito da La Niña apresentam as menores médias, considerando as defasagens de dois a quatro meses. Essa diferença pode sugerir mecanismos diferenciados das teleconexões entre o Pacífico Equatorial e a região de Maringá, para os eventos de El Niño e de La Niña. 
Anteriormente, demonstrou-se que o mês de novembro é um dos meses mais influenciado pelas anomalias de chuva, na fase quente do evento. Durante a primavera dos eventos frios, neste mesmo mês, prevalecem as anomalias secas com magnitudes máximas (GRIMM et al., 1998).

Tabela 2 - Estatísticas descritivas das anomalias de chuvas considerando os meses sob influência do El Niño. N: número de casos, $\mu$ : média, $\sigma$ : desvio padrão. O valor-p apresentado refere-se ao teste K-S Lilliefors (nível de significância $\alpha$ : 0,05). R: rejeitada, A: aceita.

\begin{tabular}{|c|c|c|c|c|c|c|}
\hline El Niño & Defasagem & $\mathbf{N}$ & M & $\sigma$ & Valor-p & Normalidade \\
\hline \multirow{5}{*}{$\begin{array}{c}\text { ATSM } \geq 0,5^{\circ} \mathrm{C} \\
\text { (todos os eventos) }\end{array}$} & 0 & 125 & 0,287 & 1,146 & 0,042 & $\mathrm{R}$ \\
\hline & 1 & 126 & 0,268 & 1,117 & 0,018 & $\bar{R}$ \\
\hline & 2 & 127 & 0,234 & 1,084 & 0,001 & $\bar{R}$ \\
\hline & 3 & 128 & 0,292 & 1,095 & 0,023 & $\mathrm{R}$ \\
\hline & 4 & 128 & 0,223 & 1,105 & 0,001 & $\bar{R}$ \\
\hline \multirow{5}{*}{$\begin{array}{c}\mathrm{ATSM} \geq 1^{\circ} \mathrm{C} \\
\text { (moderados e fortes) }\end{array}$} & 0 & 65 & 0,563 & 1,266 & 0,381 & $A$ \\
\hline & 1 & 65 & 0,480 & 1,247 & 0,081 & $\bar{A}$ \\
\hline & 2 & 65 & 0,477 & 1,263 & 0,006 & $\mathrm{R}$ \\
\hline & 3 & 65 & 0,323 & 1,092 & 0,006 & $\mathrm{R}$ \\
\hline & 4 & 65 & 0,372 & 1,129 & 0,011 & $\bar{R}$ \\
\hline
\end{tabular}

Tabela 3 - Estatísticas descritivas das anomalias de chuvas considerando os meses sob influência do La Niña. N: número de casos, $\mu$ : média, $\sigma$ : desvio padrão. O valor-p apresentado refere-se ao teste K-S Lilliefors (nível de significância a: 0,05). R: rejeitada, A: aceita.

\begin{tabular}{|c|c|c|c|c|c|c|}
\hline La Niña & Defasagem & $\mathbf{N}$ & M & $\sigma$ & Valor-p & Normalidade \\
\hline \multirow{5}{*}{$\begin{array}{c}\text { ATSM } \leq-0,5^{\circ} \mathrm{C} \\
\text { (todos os eventos) }\end{array}$} & 0 & 124 & $-0,098$ & 0,880 & 0,006 & $\mathrm{R}$ \\
\hline & 1 & 123 & $-0,138$ & 0,879 & 0,004 & $\bar{R}$ \\
\hline & 2 & 122 & $-0,141$ & 0,905 & 0,002 & $\mathrm{R}$ \\
\hline & 3 & 121 & $-0,144$ & 0,861 & 0,018 & $\mathrm{R}$ \\
\hline & 4 & 120 & $-0,155$ & 0,862 & 0,007 & $\mathrm{R}$ \\
\hline \multirow{5}{*}{$\begin{array}{l}\text { ATSM } \leq-1^{\circ} \mathrm{C} \\
\text { (moderados e } \\
\text { fortes) }\end{array}$} & 0 & 54 & $-0,176$ & 0,851 & 0,050 & $\bar{A}$ \\
\hline & 1 & 54 & $-0,202$ & 0,861 & 0,031 & $\mathrm{R}$ \\
\hline & 2 & 54 & $-0,208$ & 0,807 & 0,127 & $\bar{A}$ \\
\hline & 3 & 54 & $-0,242$ & 0,776 & 0,256 & $A$ \\
\hline & 4 & 54 & $-0,171$ & 0,738 & 0,553 & $\bar{A}$ \\
\hline
\end{tabular}


Tabela 4 - Estatísticas descritivas das anomalias de chuvas considerando os meses "normais", sem influência do ENOS. N: número de casos, $\mu$ : média, $\sigma$ : desvio padrão. O valor-p apresentado refere-se ao teste K-S Lilliefors (nível de significância $\alpha$ : 0,05). R: rejeitada, A: aceita.

\begin{tabular}{c|c|c|c|c|c|c} 
Meses "normais" & Defasagem & $\mathbf{N}$ & $\mathbf{M}$ & $\boldsymbol{\sigma}$ & Valor-p & Normalidade \\
\hline \multirow{4}{*}{$\begin{array}{c}-0,5^{\circ} \mathrm{C}<\mathrm{ATSM} \\
<0,5^{\circ} \mathrm{C}\end{array}$} & 1 & 195 & $-0,122$ & 0,906 & $1,89 \cdot 10^{-7}$ & $\mathrm{R}$ \\
\cline { 2 - 7 } & 2 & 195 & $-0,086$ & 0,932 & $1,30 \cdot 10^{-6}$ & $\mathrm{R}$ \\
\cline { 2 - 7 } & 3 & 195 & $-0,101$ & 0,950 & $1,04 \cdot 10^{-8}$ & $\mathrm{R}$ \\
\cline { 2 - 7 } & 4 & 196 & $-0,050$ & 0,956 & $1,99 \cdot 10^{-6}$ & $\mathrm{R}$ \\
\hline \multirow{3}{*}{$-1^{\circ} \mathrm{C}<\mathrm{ATSM}<1^{\circ} \mathrm{C}$} & 0 & 325 & $-0,083$ & 0,906 & $5,05 \cdot 10^{-10}$ & $\mathrm{R}$ \\
\cline { 2 - 7 } & 1 & 325 & $-0,062$ & 0,920 & $9,27 \cdot 10^{-10}$ & $\mathrm{R}$ \\
\cline { 2 - 7 } & 2 & 325 & $-0,060$ & 0,924 & $1,11 \cdot 10^{-8}$ & $\mathrm{R}$ \\
\cline { 2 - 7 } & 3 & 325 & $-0,024$ & 0,983 & $5,47 \cdot 10^{-10}$ & $\mathrm{R}$ \\
\cline { 2 - 7 } & 4 & 325 & $-0,045$ & 0,978 & $3,36 \cdot 10^{-10}$ & $\mathrm{R}$ \\
\hline
\end{tabular}

Fonte - Os autores.

Os testes de normalidade mostraram que a maioria dos grupos avaliados possui distribuição nãonormal (Tabelas 2, 3 e 4), levando ao uso dos testes de diferença entre medianas. Independente da defasagem temporal (entre zero e quatro meses) e da intensidade dos eventos (considerando os fracos ou não), os testes de diferença entre medianas forneceram resultados semelhantes.

Com base no teste de Mann-Whitney e com uso de nível de significância de 0,05, pode-se afirmar que os meses sob influência do El Niño possuem medianas superiores às medianas dos meses normais e dos meses sob influência da La Niña (Tabelas 5 e 6). Por outro lado, para o período analisado não há indícios para se afirmar que as anomalias de chuva sejam menores em meses sob influência do La Niña, quando comparados com os meses "normais".

Tabela 5 - Testes de medianas para eventos ENOS (módulo de ATSM $\geq 0,5^{\circ} \mathrm{C}$ ). Nível de significância a: 0,05 .

\begin{tabular}{|c|c|c|c|c|c|c|}
\hline Defasagem & Grupo 1 & Grupo 2 & Mediana 1 & Mediana 2 & $\begin{array}{c}\text { Mann-Whitney } \\
\text { (valor p) }\end{array}$ & Medianas \\
\hline 0 & \multirow{5}{*}{ El Niño } & \multirow{5}{*}{ Normais } & 0,084 & $-0,305$ & 0,0013 & $\neq$ \\
\hline 1 & & & 0,077 & $-0,298$ & 0,0040 & $\neq$ \\
\hline 2 & & & 0,049 & $-0,298$ & 0,0126 & $\neq$ \\
\hline 3 & & & 0,077 & $-0,325$ & 0,0007 & $\neq$ \\
\hline 4 & & & $-0,03$ & $-0,278$ & 0,0337 & $\neq$ \\
\hline 0 & \multirow{2}{*}{ La Niña } & \multirow{2}{*}{ Normais } & $-0,279$ & $-0,305$ & 0,71 & $=$ \\
\hline 1 & & & $-0,310$ & $-0,298$ & 0,69 & $=$ \\
\hline
\end{tabular}




\begin{tabular}{|c|c|c|c|c|c|c|}
\hline 2 & & & $-0,309$ & $-0,298$ & 0,45 & $=$ \\
\hline 3 & & & $-0,285$ & $-0,325$ & 0,84 & $=$ \\
\hline 4 & & & $-0,296$ & $-0,278$ & 0,36 & $=$ \\
\hline 0 & \multirow{5}{*}{ El Niño } & \multirow{5}{*}{ La Niña } & 0,084 & $-0,279$ & 0,0093 & $\neq$ \\
\hline 1 & & & 0,077 & $-0,310$ & 0,0036 & $\neq$ \\
\hline 2 & & & 0,049 & $-0,309$ & 0,0037 & $\neq$ \\
\hline 3 & & & 0,077 & $-0,285$ & 0,0011 & $\neq$ \\
\hline 4 & & & $-0,03$ & $-0,296$ & 0,0060 & $\neq$ \\
\hline
\end{tabular}

Fonte - Os autores.

Tabela 6 - Testes de medianas para eventos ENOS (módulo de ATSM $\geq 1^{\circ} \mathrm{C}$ ). Nível de significância $\alpha$ : 0,05 .

\begin{tabular}{|c|c|c|c|c|c|c|}
\hline Defasagem & Grupo 1 & Grupo 2 & Mediana 1 & Mediana 2 & $\begin{array}{c}\text { Mann-Whitney } \\
\text { (valor } p)\end{array}$ & Medianas \\
\hline 0 & \multirow{5}{*}{ El Niño } & \multirow{5}{*}{ Normais } & 0,489 & $-0,289$ & $8,84 \cdot 10^{-5}$ & $\neq$ \\
\hline 1 & & & 0,311 & $-0,262$ & 0,0009 & $\neq$ \\
\hline 2 & & & 0,075 & $-0,251$ & $7,27 \cdot 10^{-5}$ & $\neq$ \\
\hline 3 & & & $-0,008$ & $-0,249$ & 0,0170 & $\neq$ \\
\hline 4 & & & 0,062 & $-0,266$ & 0,0061 & $\neq$ \\
\hline 0 & \multirow{5}{*}{ La Niña } & \multirow{5}{*}{ Normais } & $-0,343$ & $-0,289$ & 0,56 & $=$ \\
\hline 1 & & & $-0,343$ & $-0,262$ & 0,33 & $=$ \\
\hline 2 & & & $-0,318$ & $-0,251$ & 0,38 & $=$ \\
\hline 3 & & & $-0,343$ & $-0,249$ & 0,18 & $=$ \\
\hline 4 & & & $-0,284$ & $-0,266$ & 0,62 & $=$ \\
\hline 0 & \multirow{5}{*}{ El Niño } & \multirow{5}{*}{ La Niña } & 0,489 & $-0,343$ & 0,0003 & $\neq$ \\
\hline 1 & & & 0,311 & $-0,343$ & 0,0020 & $\neq$ \\
\hline 2 & & & 0,075 & $-0,318$ & 0,0028 & $\neq$ \\
\hline 3 & & & $-0,008$ & $-0,343$ & 0,0052 & $\neq$ \\
\hline 4 & & & 0,062 & $-0,284$ & 0,0151 & $\neq$ \\
\hline
\end{tabular}

\section{CONSIDERAÇÕES FINAIS}

Maringá é um município com pluviosidade média alta e distribuição relativamente regular deste elemento ao longo do ano, frente à sua localização em área de transição climática entre o clima tropical e o subtropical. Há variabilidade pluviométrica pouco elevada na série considerada, e circulação atmosférica provavelmente influenciada por fenômenos regionais e extrarregionais como as teleconexões com o Oceano Pacífico. As principais conclusões foram:

- A correlação de Pearson realizada para toda a série resultou em baixo poder explicativo das anomalias de chuvas local pelo IOS, no entanto, a mesma correlação feita mês a mês, 
demonstrou que a influência na precipitação é mais relevante em abril, maio, setembro, novembro e dezembro, ou seja, em meses representativos do outono e da primavera. Resultados similares foram obtidos pelo coeficiente de correlação de Kendall;

- Os maiores valores de correlação apontam para uma defasagem temporal desta relação de dois a três meses, sendo significativos entre um e cinco meses para a correlação de Pearson, e de zero a quatro meses, para o coeficiente de correlação de Kendall;

- As correlações são mais significativas quando se considera apenas os eventos dentro da série que tiveram ocorrência de ENOS. De 1980 a 2016, quatro anos estiveram sob condições normais, sendo que nos demais houve 22 eventos El Niño e 16 eventos La Niña. As médias das anomalias de chuvas indicaram que os meses sob influência de El Niño possuem chuvas mais abundantes, enquanto os meses sob influência de La Niña, possuem menor quantidade de chuva;

- Quanto mais intenso o evento ENOS (moderado e forte), maior é a relação entre as variáveis, portanto, maior a diferença de precipitação com relação aos meses de neutralidade. No entanto, meses de ocorrência de La Niña possuem médias de anomalias de chuva próximas aos dos meses com condições neutras, não sendo possível concluir o inverso do observado para o El Niño.

\section{REFERÊNCIAS}

BERLATO, M. A.; FONTANA, D. C. El Niño e La Niña: impactos no clima, na vegetação e na agricultura do Rio Grande do Sul; aplicações de previsões climáticas na agricultura. Porto Alegre: Editora da UFRGS, 2003. 110 p.

CAVALCANTI, I. F. de A. Episódios de EI Niño/Oscilação Sul durante a década de 1986 a 1996 e suas influências sobre o Brasil. Climanálise, São José dos Campos, 1996. Disponível em: <h ttp://climanalise.cptec.inpe.br/ rclimanl/boletim/cliesp10a/nino.html>. Acesso em: 01 de fevereiro de 2018.

CPC/NOAA. Climate Prediction Center/ National Oceanic and Atmospheric Administration. Monthly Niño 3.4 index. Disponível em: <http://origin.cpc.ncep.noaa.gov/products/analysis_mo nitoring/ensostuff/detrend.nino34.ascii.txt>. Acesso em: 05 de dezembro de 2017.

CPC-NOAA. Climate Prediction Center/ National Oceanic and Atmospheric Administration. Southern Oscillation Index (SOI). Disponível em: <https://www.ncdc.noaa.gov/teleconnection

s/enso/indicators/soi/>. Acesso em: 05 de dezembro de 2017.

CUNHA, G. R. da; DALMAGO, G. A.; ESTEFANEL, V.; PASINATO, A.; MOREIRA, M. B. EI Niño, oscilação do sul e seus impactos sobre as culturas de trigo e de cevada no Brasil. Passo Fundo: Embrapa Trigo, 2000. 44 p. (Embrapa Trigo. Boletim de Pesquisa, 9). Disponível em: $<$ https://ainfo.cnptia.embrapa.br/digital/bitstream/item/121360/1/FL-07288.pdf>. Acesso em: 03 de janeiro de 2017.

DEFFUNE, G. Clima e uso da terra no Norte e Noroeste do Paraná - 1975/1986: subsídios ao planejamento regional. Dissertação (Mestrado em Geografia) - São Paulo: USP, 1990.

ECPM/INMET. Estação Climatológica Principal de Maringá/ Instituto Nacional de Meteorologia. Disponível em: <http://www.inmet.gov.br/sim/sonabra/dspDadosCodigo.php?ODM3Njc=>. Acesso em: 05 de dezembro de 2017. 
FERREIRA, J. H. D. Relação de parâmetros meteorológicos do Estado do Paraná associados à anomalia da temperatura da superfície do mar no Pacífico Equatorial. Dissertação (Mestrado em Geografia) - Maringá: UEM, 2000.

GALVANI, E.; PEREIRA, A. R.; KLOSOWSKI, E. S. Relações entre o índice de oscilação sul (IOS) e o total de chuva em Maringá-PR. Revista Acta Scientiarum, v. 20, n. 4, p. 531-535. 1998. Disponível em: <http://periodicos.uem.br/ojs/index.php/ActaSciTechnol/article/view/3124 >. Acesso em: 06 de maio de 2016.

GRIMM, A. M.; FERRAZ, S. E. T.; GOMES, J. Precipitation anomalies in Southern Brazil associated with EI Niño and La Niña events. Journal of Climate, 11, 1998, p. 2863-2880. Disponível em: <https://journals.ametsoc.org/doi/abs/10.1175/1520 0442(1998)011\%3C2863\% https://doi.org/10.1175/1520-0442(1998)011<2863:PAISBA>2.0.CO;2

APAISBA\%3E2.0.CO\%3B2>. Acesso em: 07 de fevereiro de 2018.

GRIMM, A. M.; BARROS, V. R.; DOYLE, M. E. Climate variability in Southern South America associated with El Niño and La Niña events. Journal of Climate, v. 13, 2000, p. 36-58. Disponível em: <https://journals.ametsoc.org/doi/pdf/10.1175/15200442\%282000\%29013\%3C https://doi.org/10.1175/1520-0442(2000)013<0035:CVISSA>2.0.CO;2

0035\%3ACVISSA\%3E2.0.CO\%3B2>. Acesso em: 18 de janeiro de 2018.

GRIMM, A. M. Clima da Região Sul do Brasil. In: CAVALCANTI, I. F. de A.; FERREIRA, N. J.; SILVA, M. G. A. J. da; DIAS, M. A. F. da. (Org.). Tempo e clima no Brasil. São Paulo: Oficina de Textos, 2009. p. 259-275.

. Variabilidade interanual do clima no Brasil. In: CAVALCANTI, I. F. de A.; FERREIRA, N. J.; SILVA, M. G. A. J. da; DIAS, M. A. F. da. (Org.). Tempo e clima no Brasil. São Paulo: Oficina de Textos, 2009. p. 353-374.

IBGE. Divisão do Brasil em mesorregiões e microrregiões geográficas. Rio de Janeiro: IBGE, 1990. 135 p. Disponível em: <https://biblioteca.ibge.gov.br/visualizacao/monografias/GE

BIS\%20-\%20RJ/DRB/Divisao\%20regional_v01.pdf>. Acesso em: 08 de agosto de 2017.

KOUSKY, V. E.; KAGANO, M. T.; CAVALCANTI, I. F. de A. A review of the Southern Oscillation: oceanic-atmospheric circulation changes and related rainfall anomalies. Tellus, 36A, 1984, p. 490-504. Disponível em: <http://onlinelibrary.wiley.com/doi/10.1111/j.16000870.1984.tb00264.x/abstract>. Acesso em: 08 de fevereiro de 2018. https://doi.org/10.1111/j.1600-0870.1984.tb00264.x

MAACK, R. Geografia física do estado do Paraná. 4. ed. Ponta Grossa, PR: Editora UEPG, 2012. 526 p.

MONTEIRO, C. A. de F. A frente polar atlântica e as chuvas de inverno na fachada sul-oriental do Brasil: contribuição metodológica à análise rítmica dos tipos de tempo no Brasil. São Paulo, SP: USP/IG, 1969. 68 p. 
NERY, J. T.; CARFAN, A. C.; ANDRADE, A. R. de. Correlação da precipitação e da vazão na bacia do Ivaí, Paraná. In: SIMPÓSIO BRASILEIRO DE RECURSOS HÍDRICOS, 15. , 2003, Curitiba. Anais... São Paulo: Sociedade Brasileira dos Recursos Hídricos, 2003, v. 1, p. 230242. Disponível em: <http://www.ourinhos.unesp.br/Home/Pesquisa/GruposdeEstudo/Clima/Si

mposios/077.pdf>. Acesso em: 06 de maio de 2016.

NIMER, E. Climatologia do Brasil. 2. ed. Rio de Janeiro, RJ: Fundação IBGE, 1989. 421 p.

OLIVEIRA, G. S. de. El Niño/ La Niña. Meio ambiente e ciências atmosféricas. São José dos Campos: INPE, 2005. CD-ROM. Disponível em: <http://mtc-m16b.sid.inpe.br/col/sid.inpe.br/iris

@1915/2005/11.08.13.01/doc/05_El_nino.pdf>. Acesso em: 23 de setembro de 2016.

RAO, V. B.; HADA, K. Characteristics of rainfall over Brazil: anual variations and connections with the Southern Oscillation. Theoretical and Applied Climatology, 42, 1990. p. 81-90. Disponível em: <https://link.springer.com/content/pdf/10.1007\%2FBF00868215.pdf>. Acesso em: 19 de janeiro de 2018. https://doi.org/10.1007/BF00868215

R CORE TEAM. R: A language and environment for statistical computing. R Foundation for Statistical Computing, Vienna, Austria. 2017. Disponível em: <https://www.R-project.org/>. Acesso em: 02 de janeiro de 2018.

ROPELEWSKI, C. F.; HALPERT, M. S. Global and regional scale precipitation patterns associated with the El Niño/Southern Oscillation. Monthly Weather Review, v. 115, 1987, p. 1606-1626. Disponível em: <https://journals.ametsoc.org/doi/pdf/10.1175/1520$0493 \% 281987 \%$ https://doi.org/10.1175/1520-0493(1987)115<1606:GARSPP>2.0.CO;2

29115\%3C1606\%3AGARSPP\%3E2.0.CO\%3B2>. Acesso em: 08 de fevereiro de 2018.

SANTOS, J. W. M. C. O clima urbano de Maringá: ensaio metodológico para cidades de porte médio e pequeno. Dissertação (Mestrado em Geografia) - São Paulo: USP, 1996.

SILVEIRA, L. M. da. Análise rítmica dos tipos de tempo no Norte do Paraná, aplicada ao clima local de Maringá-PR. Tese (Doutorado em Geografia Física) - São Paulo: USP, 2003.

TEIXEIRA, N. F.; MARTÍN-VIDE, J. Influência do ENOS na distribuição das precipitações no Estado do Paraná, Brasil. Revista Brasileira de Climatologia, ano 9, v. 13, jan-jul. 2013. p. 103113. Disponível em: <http://revistas.ufpr.br/revistaabclima/article/view/30484/21509>. Acesso em: 07 de setembro de 2016.

TRENBERTH, K. E. The definition of El Niño. Bulletin of the American Meteorological Society. 1997. p. 2771-2777. Disponível em: <http://journals.ametsoc.org/doi/pdf/10.1175/1520 https://doi.org/10.1175/1520-0477(1997)078<2771:TDOENO>2.0.CO;2

Recebido em: 20/02/2018

Aceito para publicação em: 01/11/2018 